\title{
A TWO-CHROMOPHORE MODEL FOR TWO-PHOTON CIRCULAR DICHROISM
}

\author{
D.L. ANDREWS \\ Department of Chemistry, University College London, Gower Street, London WCIE 6BT, UK
}

Received 5 April 1976

\begin{abstract}
A discussion of two-photon circular dichroism due to dissymmetrically placed chromophores is presented. Using quantum electrodynamics, expressions are derived for the differential rates of absorption associated with an electronic transition which is electric dipole forbidden to a one-photon interaction. A complex field formulation enables effective interaction operators to be derived which are formally equivalent to those arising in the theory of induced circular dichroism. For nonequivalent chromophores, it is shown that a coupling mechanism provides the necessary chiral discrimination, whilst for equivalent chromophores there is an additional contribution from an interference term which becomes more important as the chromophore separation is decreased.
\end{abstract}

\section{Introduction}

With the development of high intensity laser sources, there has recently been much interest in non-linear effects such as two-photon absorption by atoms and molecules. One of the interesting features is that the rate of absorption for chiral molecules is dependent upon the handedness of the incident light. This effect is known as two-photon circular dichroism, and has recently been discussed by Power [1] and Tinoco [2].

Although the exhibition of circular dichroism is dependent upon an overall dissymmetry in the molecule, the electronic absorption bands are frequently associated with transitions in individual chromophores which are not inherently chiral. Consequently it is instructive to consider a model for two-photon absorption in which chiral discrimination is provided by a pair of achiral chromophores placed dissymmetrically relative to each other, and in which the electronic transition of interest is confined to one of these chromophores. This model is therefore adopted for the theory below, and since we are concerned with two-photon absorption, it is assumed that one-photon interactions are electric dipole forbidden by virtue of the local symmetry.

In the following account, we begin with a general development of the theory within the framework of quantum. electrodynamics. A complex field formulation is employed which facilitates dealing with circularly polarised photons, and which enables a formal equivalence to be demonstrated between the effective interaction operators used here and those which arise in the theory of induced circular dichroism. The theory is then applied to the two specific cases where the chromophore pairs are either chemically equivalent or non-equivalent, and the dependence of the differential absorption rate upon the chromophore separation is examined. Finally, the practical likelihood of observing two-photon circular dichroism is discussed, on the basis of the results obtained for the two-chromophore model.

\section{Theory}

We may begin by writing down the hamiltonian for the system as

$$
H=H_{\text {molecules }}+H_{\text {radiation }}+H_{\text {interaction }}
$$

where it is assumed that the molecular hamiltonians are known, and the radiation hamiltonian is given by 


$$
\dot{H}_{\mathrm{rad}}=\frac{1}{8 \pi} \int\left(e^{12}+b^{2}\right) \mathrm{d}^{3} r
$$

in which $e^{\perp}$ and $b$ are the electric and magnetic field operators, respectively. Since we shall be dealing with only circularly polarised photons, it is convenient to employ complex fields in writing down the interaction hamiltonian. Following Power and Thirunamachandran [3], we define the following field operators:

$$
\begin{aligned}
& d=p+i m, \\
& f=e^{\perp}+\mathrm{i} b,
\end{aligned}
$$

and their hermitian conjugates $d^{\dagger}, f^{\dagger}$. Here $p$ and $m$ are the electric polarisation and magnetisation fields respectively. The interaction hamiltonian in dipole approximation is

$$
H_{\mathrm{int}}=-\frac{1}{2} \sum_{\xi}\left[d^{\frac{1}{\dagger}}(\xi) \cdot f\left(R_{\xi}\right)+d(\xi) \cdot f^{\dagger}\left(R_{\xi}\right)\right],
$$

where the sum is over all molecules $\xi$ at position $\boldsymbol{R}_{\xi}$. The complex radiation fields may now be expanded in the usual way in terms of annihilation $a^{(\lambda)}(k)$ and creation $a^{(\lambda)^{\dagger}}(k)$ operators for the mode of wavevector $k$ and polarisation $\lambda$;

$$
\begin{aligned}
& f(r)=\sum_{k}\left(8 \pi \frac{\hbar c k}{V}\right)^{1 / 2} \mathrm{i}\left[e^{(\mathrm{L})}(k) a^{(\mathrm{L})}(k) \mathrm{e}^{\mathrm{i} k \cdot r}-\bar{e}^{(\mathrm{R})}(k) a^{(\mathrm{R}) \dagger}(k) \mathrm{e}^{-\mathrm{i} k \cdot r}\right], \\
& f^{\dagger}(r)=\sum_{k}\left(8 \pi \frac{\hbar c k}{V}\right)^{1 / 2} \mathrm{i}\left[e^{(\mathrm{R})}(k) a^{(\mathrm{R})}(k) \mathrm{e}^{\left.\mathrm{i} k \cdot r^{-}-\bar{e}^{(\mathrm{L})}(k) a^{(\mathrm{L}) \dot{\mathrm{i}}}(k) \mathrm{e}^{-\mathrm{i} k \cdot r}\right] .}\right.
\end{aligned}
$$

Since the overall process involves a change of two photons, only even orders in $H_{\text {int }}$ contribute to the matrix element, which is given by

$$
M_{f i}=\left\langle f\left|H_{\text {int }} \frac{1}{E-H_{0}} H_{\text {int }}+H_{\text {int }} \frac{1}{E-I_{0}} H_{\text {int }} \frac{1}{E-H_{0}} H_{\text {int }} \frac{1}{E-I I_{0}} H_{\text {int }}+\ldots\right| i\right\rangle,
$$

where $|i\rangle$ is the initial state of the system and $|f\rangle$ the final state. The second order contribution, which we shall write as $M_{f i}^{(2)}$, corresponds to a two-photon process involving only one chromophore (A); the fourth order contribution $M_{f i}^{(4)}$ involves two chiomophores ( $\mathrm{A}$ and $\mathrm{B}$ ) dynamically coupled by a virtual photon. As the former result is easily evaluated, we concentrate for the present upon the coupled term.

Let us suppose that both the incident photons are derived from the same beam, of wavevector $k$ and polarisation vector $e^{(\mathrm{L}, \mathrm{R})}$, and that the two chromophores are coupled by a virtual photon of wavevector $\mathrm{k}$ and polarisation vector $e^{(\epsilon)}$. The calculation of the coupled contribution to the matrix element is facilitated by construction of a full set of time-ordered graphs of which fig. 1(a) is an example. For any given polarisations of the photons involved,

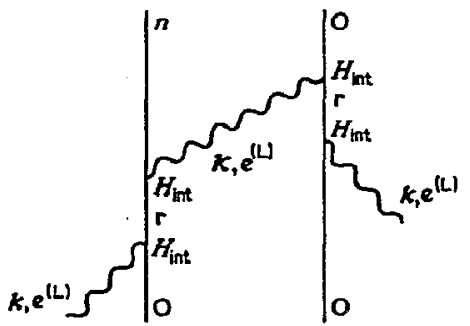

A

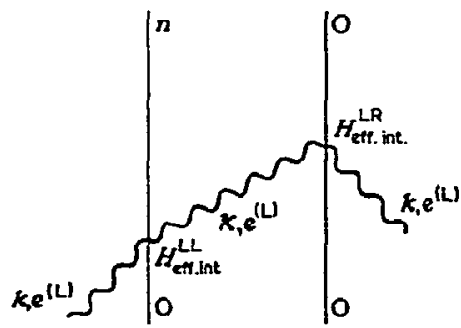

A

(a)

Fig. 1. Typical time-ordered diagrams for two-photon absorption involving two chromophores. 
there are 24 such diagrams to be taken into account, each representing different time sequences; twelve have the virtual photon propagating from $A$ to $B$, as shown, and the other twelve the reverse. If we add together the twelve belonging to the first set, the summed matrix element contracts to a form which is obtainable from a single timeordered diagram such as that shown in fig. 1 (b). (Similar situations are encountered in the study of Rayleigh and Raman scattering [4].) Now there are two photons associated with each contracted vertex, and the effective interaction energy may be written as

$$
\eta_{\text {eff.int }}=\eta_{H} H_{\text {eff.int }}^{\mathrm{LL}}+\eta_{H_{\text {eff.int }}^{\mathrm{LR}}}^{\mathrm{LR}}+\eta_{H_{\text {eff.int }}^{\mathrm{RL}}}^{\mathrm{L}}+\eta_{\text {eff.int }}^{\mathrm{RR}},
$$

with the various terms given as follows;

$$
\begin{aligned}
& { }^{\eta} H_{\text {eff.int }}^{\mathrm{LL}}=-\frac{1}{4} \sum_{r_{\eta}}\left[\frac{{ }^{\eta} d_{i}^{n r} \eta_{d_{j}^{\dagger} r 0}}{E_{r 0}^{\eta}-\hbar c k}+\frac{{ }^{\eta} d_{j}^{\dagger} n r \eta_{d}^{r 0}}{E_{m}^{\eta}+\hbar c k}\right] f_{i}^{\dagger}(\kappa) f_{j}(k) \text {, }
\end{aligned}
$$

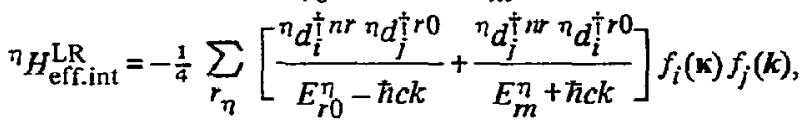

$$
\begin{aligned}
& \eta_{\mathrm{eff}_{\text {.int }}}^{\mathrm{RL}}=-\frac{1}{4} \sum_{r_{\eta}}\left[\frac{{ }^{\eta} d_{i}^{n r} \eta_{j}^{r 0}}{E_{r 0}^{\eta}-\hbar c k}+\frac{\eta_{d_{j}^{n r}}^{n r} d_{i}^{r 0}}{E_{r n}^{\eta}+\hbar c k}\right] f_{i}^{\dagger}(\mathrm{k}) f_{j}^{\dagger}(k) \text {, }
\end{aligned}
$$

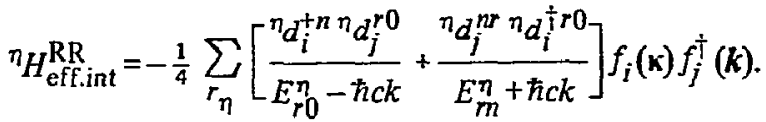

Here $\eta$ is the label for the chromophore at which the two-photon process occurs. This has ground state $|0\rangle$, summed intermediate states $|r\rangle$, and a final state $|n\rangle$ which is the ground state when $\eta$ refers to the unexcited chromophore B. From the properties of the field operators, we find that each of the four terms in eq. (9) has a simple interpretation; $H^{\lambda \mu}$ is responsible for absorption of a real photon of polarisation $e^{(\lambda)}$, and either emission of a virtual photon of polarisation $e^{(\mu)}$, or absorption of a virtual photon of polarisation $\bar{e}^{-(\mu)}$. These terms are of exactly similar form to those which arise in the theory of induced circular dichroism [5].

Now since we are here concerned with treatment of a system in which $A$ and $B$ are not inherently chiral, we are justified in taking only the leading electric dipole terms in $d, d^{\dagger}$. The interaction energy for the contracted graphs becomes quite simply

$$
{ }^{\eta} H_{\text {eff.int }}=-\sum_{r_{\eta}}\left[\frac{{ }^{\eta} \mu_{i}^{n r} \eta_{\mu_{j}^{r 0}}^{r 0}}{E_{r 0}^{\eta}-\hbar c k}+\frac{\eta_{\mu_{j}^{n r}}^{n} \eta_{\mu_{i}^{r 0}}}{E_{m}^{\eta}+\hbar c k}\right] e_{i}^{\perp}(\boldsymbol{k}) e_{j}^{\perp}(k),
$$

which may be represented as

$$
{ }^{\eta} H_{\text {eff.int }}=-\eta_{\alpha_{i j}^{n 0}(k)}^{n !}(\mathbf{k}) e_{j}^{\perp}(k) .
$$

The overall matrix element $M_{f i}^{(4)}$ for the dynamically coupled absorption process is now given by the sum of the matrix element for the graphs represented by fig. 1(b), and the corresponding matrix element for graphs in which the virtual photon passes from B to A. Assuming real wavefunctions, and summing over the polarisations and wavevector of the virtual photon, we obtain

$$
M_{f i}^{(4)}=-8 \pi^{2} \frac{\hbar c k[n(n-1)]^{1 / 2}}{V} \mathrm{~A}_{\alpha_{i m}^{n 0}}(k)^{\mathrm{B}} \alpha_{j n}^{00}(k) V_{m n}(k, R) e_{i} e_{j} \mathrm{e}^{\mathrm{i} k \cdot R},
$$

where $V$ is the volume of the quantisation box containing $n$ photons, $V_{m n}(k, R)$ is the usual retarded resonance electric-electric dipole interaction [6] given by

$$
V_{m n}(k, R)=\frac{1}{4 \pi R^{3}}\left[\left(\delta_{m n}-3 \hat{R}_{m} \hat{R}_{n}\right)(\cos k R+k R \sin k R)-\left(\delta_{m n}-\hat{R}_{m} \hat{R}_{n}\right) k^{2} R^{2} \cos k R\right],
$$


and $R$ is the vector $\overrightarrow{A B}$. The origin of the molecular coordinate system has been arbitrarily chosen to be coincident with $\mathrm{A}$.

Having completed the derivation of the result for the fourth order contribution to the matrix element for twophoton absorption, we now proceed to evaluate the rate expressions required for a description of the associated circular dichroism. From Fermi's golden rule, the required rate expression for a system of randomly oriented molecules is given by

$$
\Gamma=\frac{2 \pi}{\hbar}\left\langle\left|M_{f i}\right|^{2}\right\rangle \rho_{i}=\frac{2 \pi}{\hbar}\left\langle\left|M_{f i}^{(2)}+M_{f i}^{(4)}+\ldots\right|^{2}\right\rangle \rho_{i},
$$

where $\rho_{i}$ is the initial density of states, and the angular brackets denote a rotational average over the Euler angles relating the molecular frame to the laboratory frame. The expression for $M_{f i}^{(4)}$ is as given in eq. (16), and it is a simple matter to show that the second order term is given by

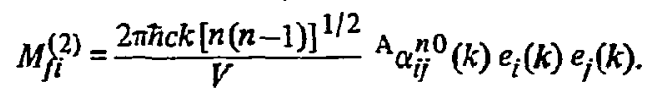

The dominant contribution to the rate obviously comes from the square of this term, and is readily shown to be

$$
{ }^{(2-2)} \Gamma(\mathrm{L} / \mathrm{R})=\frac{2 \pi}{\hbar}\left\langle\left|M_{f i}^{(2)}(\mathrm{L} / \mathrm{R})\right|^{2}\right) \rho_{i}=\frac{8 \pi^{3} \hbar c^{2} k^{2} n(n-1) \rho_{i}}{15 V^{2}}\left(3^{\mathrm{A}} \alpha_{\lambda \mu}^{n 0 \mathrm{~A}} \alpha_{\lambda \mu}^{n 0}-{ }^{\mathrm{A}} \alpha_{\lambda \lambda}^{n 0 \mathrm{~A}} \alpha_{\mu \mu}^{n 0}\right),
$$

in which the implied summation convention for repeated indices has been employed. The first correction term results from the second-fourth order cross terms in eq. (18). However, in this case, the result differs according to the handedness of the incident radiation, and we find that

$$
\begin{aligned}
& { }^{(2-4)} \Gamma(\mathrm{L})-{ }^{(2-4)} \Gamma(\mathrm{R})=\frac{-256 \mathrm{i} \pi^{4} \hbar c^{2} k^{2} n(n-1) \rho_{i}}{V^{2}} V_{\rho \sigma}(k, R) \epsilon_{y \pi r} \hat{R}_{\tau} \\
& \quad \times\left[\left(\delta_{\lambda \mu}-\hat{R}_{\lambda} \hat{R}_{\mu}\right) \mathcal{S}_{1}(k R)+\hat{R}_{\lambda} \hat{R}_{\mu} \mathcal{S}_{2}(k R)\right]\left({ }^{\mathrm{A}} \alpha_{\lambda \nu}^{n 0 \mathrm{~A}} \alpha_{\mu \rho}^{n 0 \mathrm{~B}} \alpha_{\sigma \pi}^{00}+{ }^{\mathrm{A}} \alpha_{\lambda \nu}^{n 0 \mathrm{~A}} \alpha_{\pi \rho}^{n 0 \mathrm{~B}} \alpha_{\sigma \mu}^{00}\right),
\end{aligned}
$$

where $\mathcal{I}_{1}$ and $\mathcal{S}_{2}$ are functions which arise from the rotational averaging. These have been discussed in another context [4], and are given by

$$
\begin{aligned}
& \mathcal{S}_{1}(a)=\frac{1}{8 \mathrm{i}}\left(\frac{\cos a}{a}-\frac{2 \sin a}{a^{2}}-\frac{3 \cos a}{a^{3}}+\frac{3 \sin a}{a^{4}}\right), \\
& \Im_{2}(a)=\frac{1}{2 \mathrm{i}}\left(\frac{\sin a}{a^{2}}+\frac{3 \cos a}{a^{3}}-\frac{3 \sin a}{a^{4}}\right) .
\end{aligned}
$$

It is now convenient to define a dimensionless differential rate as

$$
D=[\Gamma(L)-\Gamma(R)] / \Gamma,
$$

where $\Gamma$ is the mean rate given by

$$
\Gamma=\frac{1}{2}[\Gamma(\mathrm{L})+\Gamma(\mathrm{R})] \text {. }
$$

The quantity $D$ provides a more absolute measure of the circular dichroism than the rate difference itself, since the former is clearly independent of the intensity and statistical properties of the incident radiation [1]. From the above considerations, we find that the leading term in the expression for the differential rate is as follows;

$$
D \simeq-480 \mathrm{i} \pi V_{\rho \sigma}(k, R) \epsilon_{\nu \pi T} \hat{R}_{\tau} \frac{\left[\left(\delta_{\lambda \mu}-\hat{R}_{\lambda} \hat{R}_{\mu}\right) \mathcal{S}_{1}(k R)+\hat{R}_{\lambda} \hat{R}_{\mu} \mathcal{G}_{2}(k R)\right]{ }^{\mathrm{A}} \alpha_{\lambda \nu}^{n 0}\left({ }^{\mathrm{A}} \alpha_{\mu \rho}^{n 0 \mathrm{~B}} \alpha_{\sigma \pi}^{00}+\mathrm{A}_{\alpha_{\mu \rho}}^{n 0 \mathrm{~B}} \alpha_{\sigma \mu}^{00}\right)}{3 \mathrm{~A}_{\alpha}{ }_{\lambda \mu}^{n 0 \mathrm{~A}} \alpha_{\lambda \mu}^{n 0}-\mathrm{A}_{\alpha_{\lambda \lambda}}^{n 0 \mathrm{~A}} \alpha_{\mu \mu}^{n 0}}
$$


Whilst this expression holds true for any interchromophore distance $R$, it is interesting to note the asymptotic behaviour for the regions in which $R$ is either large or small compared to the reduced wavelength $\lambda(=1 / k)$. The first case corresponds to $k R \gg 1$ and the second ro $k R \ll 1$.

In the first case, it may be shown that $V(k, R)$ and $\mathcal{S}_{1}(k R)$ both tend to a $(\cos k R) / k R$ dependence, whilst the $9_{2}$ term can be ignored. Hence $D$ falls off with a modulated inverse square dependence upon $R$ in this region.

However, in the physically more interesting region with $k R \ll 1$, we have

$$
\begin{aligned}
& \mathcal{S}_{1}(a) \simeq \mathcal{O}_{2}(a) \simeq \mathrm{i} a / 30, \\
& V_{\rho \sigma}(k, R) \simeq \frac{1}{4 \pi R^{3}}\left(\delta_{\rho \sigma}-3 \hat{R}_{\rho} \hat{R}_{\sigma}\right) .
\end{aligned}
$$

Hence, in this region, the result of eq. (26) simplifies to the form

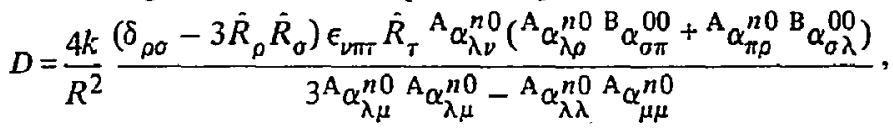

and there is still an inverse square dependence upon $R$. This asymptotic behaviour is directly analogous to that of the Raman circular intensity differential for non-identical achiral chromophores [4].

The above theory may readily be extended to the case in which A and B are chemically equivalent, by adoption of appropriate excited states. The stationary states for the molecular system in which only one centre is excited are now

$$
\left|n_{ \pm}\right\rangle=\frac{1}{2^{1 / 2}}\left(\left|n_{\mathrm{A}}\right\rangle\left|0_{\mathrm{B}}\right\rangle \pm\left|0_{\mathrm{A}}\right\rangle\left|n_{\mathrm{B}}\right\rangle\right)
$$

and the matrix elements have to be reformulated to give $M_{\ddagger}$. The dominant rate contribution as usual comes from the square of the second order term, and is given by

$$
\begin{aligned}
& { }^{(2-2)} \Gamma_{ \pm}(\mathrm{L}) \simeq{ }^{(2-2)} \Gamma_{ \pm}(\mathrm{R}) \simeq \frac{4 \pi^{3} \hbar c^{2} k^{2} n(n-1) \rho_{i}}{15 V^{2}} \\
& \quad \times\left[3\left({ }^{\mathrm{A}} \alpha_{\lambda \mu}^{n 0} \pm{ }^{\mathrm{B}} \alpha_{\lambda \mu}^{n 0}\right)\left({ }^{\mathrm{A}} \alpha_{\lambda \mu}^{n 0} \pm{ }^{\mathrm{B}} \alpha_{\lambda \mu}^{n 0}\right)-\left({ }^{\mathrm{A}} \alpha_{\lambda \lambda}^{n 0} \pm{ }^{\mathrm{B}} \alpha_{\lambda \lambda}^{n 0}\right)\left({ }^{\mathrm{A}} \alpha_{\mu \mu}^{n 0} \pm{ }^{\mathrm{B}} \alpha_{\mu \mu}^{n 0}\right)\right] .
\end{aligned}
$$

However, as a consequence of the equivalence of $A$ and $B$, the interference terms in ${ }^{(2-2)} \Gamma_{ \pm}$are in fact slightly different for left and right circularly polarised light. The difference in rates, which is much smaller than the mean rate of eq. (31), is

$$
\begin{aligned}
& { }^{(2-2)} \Gamma_{ \pm}(\mathrm{L})-{ }^{(2-2)} \Gamma_{ \pm}(\mathrm{R})= \pm \frac{64 \mathrm{i} \pi^{3} \hbar c^{2} k^{2} n(n-1) \rho_{i}}{V^{2}} \\
& \quad \times \epsilon_{v \pi \pi} \hat{R}_{\tau}\left[\left(\delta_{\lambda \mu}-\hat{R}_{\lambda} \hat{R}_{\mu}\right) \Im_{1}(2 k R)+\bar{R}_{\lambda} \hat{R}_{\mu} \Im_{2}(2 k R)\right]{ }^{\mathrm{A}} \alpha_{\lambda \nu}^{n 0 \mathrm{~B}} \alpha_{\mu \pi}^{n 0} .
\end{aligned}
$$

In addition to this new term, there is once again a contribution to the rate difference coming from the secondfourth order matrix element cross terms, which is as follows;

$$
\begin{aligned}
& { }^{(2-4)} \Gamma_{ \pm}(\mathrm{L})-{ }^{(2-4)} \Gamma_{ \pm}(\mathrm{R}) \\
& =\frac{-128 \mathrm{i} \pi^{4} \hbar c^{2} k^{2} n(n-1) \rho_{i}}{V^{2}} V_{\rho \sigma}(k, R) \epsilon_{\nu \pi r} \hat{R}_{\tau}\left[\left(\delta_{\lambda \mu}-\hat{R}_{\lambda} \hat{R}_{\mu}\right) 9_{1}(k R)+\hat{R}_{\lambda} \hat{R}_{\mu} g_{2}(k R)\right] \\
& \times\left[\left({ }^{\mathrm{A}} \alpha_{\lambda \nu}^{n 0 \mathrm{~A}} \alpha_{\mu \rho}^{n 0 \mathrm{~B}} \alpha_{\sigma \pi}^{00}+{ }^{\mathrm{A}} \alpha_{\lambda \nu}^{n 0 \mathrm{~A}} \alpha_{\pi \rho}^{n 0 \mathrm{~B}} \alpha_{\sigma \mu}^{00}-{ }^{\mathrm{B}} \alpha_{\lambda \nu}^{n 0 \mathrm{~B}} \alpha_{\mu \rho}^{n 0 \mathrm{~A}} \alpha_{\sigma \pi}^{00}-{ }^{\mathrm{B}} \alpha_{\lambda \nu}^{n 0 \mathrm{~B}} \alpha_{\pi \rho}^{n 0 \mathrm{~A}} \alpha_{\sigma \mu}^{00}\right)\right. \\
& \left. \pm\left({ }^{\mathrm{A}} \alpha_{\lambda \nu}^{n 0 \mathrm{~B}} \alpha_{\mu \rho}^{n 0 \mathrm{~A}} \alpha_{\sigma \pi}^{00}+{ }^{\mathrm{A}} \alpha_{\lambda \nu}^{n 0 \mathrm{~B}} \alpha_{\pi \rho}^{n 0 \mathrm{~A}} \alpha_{\sigma \mu}^{00}-{ }^{\mathrm{B}} \alpha_{\lambda \nu}^{n 0 \mathrm{~A}} \alpha_{\mu \rho}^{n 0 \mathrm{~B}} \alpha_{\sigma \pi}^{00}-{ }^{\mathrm{B}} \alpha_{\lambda \nu}^{n 0 \mathrm{~A}} \alpha_{\pi \rho}^{n 0 \mathrm{~B}} \alpha_{\sigma \mu}^{00}\right)\right] .
\end{aligned}
$$


The resulting approximate expression for $D$ therefore has as its numerator the sum of eqs. (32) and (33), and as its denominator the expression of eq. (31). In discussing these cases we must remember that as the chromophore separation $R$ increases, the difference in energy between the two states $\left|n_{ \pm}\right\rangle$will diminish until they are indistinguishable. In that case, the observed rate difference will be the sum of the $\Gamma_{+}$and $\Gamma_{-}$rate differences, which means that the contribution from eq. (32) vanishes, as does the \pm term in eq. (33).

As before, we may again examine the asymptotic behaviour for $k R \geqslant 1$ and $k R \ll 1$. In the first case, the leading contribution from eq. (33) has a modulated $R^{-2}$ dependence, and that of eq. (32) a modulated $R^{-1}$ dependence. In the second case, using the results of eqs. (27) and (28) which are applicable in this region, we obtain the usual $R^{-2}$ dependence for the contribution of eq. (33), but we find that the lower order contribution of eq. (32) is now linearly dependent upon $R$. It therefore appears that in the region $k R \ll 1$, the differential rate is approximately given by

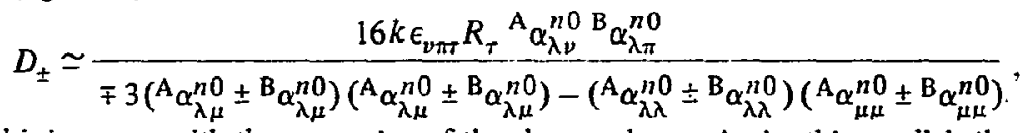

and this increases with the separation of the chromophores. Again, this parallels the well-established behaviour of the Raman circular intensity differential for identical achiral chromophores $[4,7]$.

\section{Discussion}

We may conclude with a brief discussion of the likelihood of observing two-photon circular dichroism due to dissymmetrically placed chromophores, as in the model adopted above. We shall be mainly interested in the region $k R \ll 1$, since it is here that $D$ attains its highest values. Let us deal first with the case of non-identical chromophores. Order of magnitude estimates suggest that when the separation is small, the differential ratio for the system as represented by eq. (29) may reach a value in the region $10^{-3}$ to $10^{-4}$, a range similar to that for one-centre two-photon circular dichroism [1], and not much smaller than the corresponding value for ordinary one-photon circular dichroism, $\left(10^{-2}\right.$ to $\left.10^{-3}\right)$. Perhaps more interesting, however, is the result eq. (34) for the identical chromophore situation. Here, it would appear that values just as large as those for ordinary circular dichroism may indeed arise. In this case, because of the slightly different energies of the two states $\left|n_{ \pm}\right\rangle$, two-photon absorption should be observed at two closely similar frequencies, circular dichroism of equal magnitude but opposite sign being associated with each component of the doublet.

\section{Acknowledgement}

I am grateful to Dr. T. Thirunamachandran for his generous help and many useful discussions. I would also like to thank Prof. E.A. Power for valuable comments, and the Science Research Council for the award of a Studentship.

\section{References}

[1] E.A. Power, J. Chem. Phys. 63 (1975) 1348.

[2] I. Tinoco Jnt., J. Chem. Plyys. 62 (1975) 1006.

[3] E.A. Power and T. Thirunamachandran, J. Chem. Phys. 60 (1974) 3695.

[4] T. Thirunamachandran and D.L. Andrews, to be published.

[5] D.P. Craig, E.A. Power and T. Thirunamachandran, Chem. Phys. Letters 27 (1974) 149; Proc. Roy. Soc. A348 (1976) 19.

[6] R.R. McLone and E.A. Power, Mathematika 11 (1964) 91.

[7] L.D. Barron and A.D. Buckingham, J. Am. Chem. Soc. 96 (1974) 4769. 